\title{
Das toponymische Erbe der Habsburgermonarchie in Galizien*
}

\author{
WOJCIECH WŁOSKOWICZ \\ Institute of the Polish Language, Polish Academy of Sciences, \\ al. Mickiewicza 31, PL-31-120 Kraków \\ E-mail:wloskowicz@gmail.com
}

(Received: 20 April 2018; accepted: 8 June 2018)

\begin{abstract}
The paper discusses the influence of the Habsburg topographic surveys and cartography on the toponymic landscape of the former crown land of Galicia. Publicly available maps have had a great impact on the geographical names used both by locals and non-locals. The Habsburg toponymic policy was characterized by non-Germanisation of already existing Galician toponyms. The Habsburg toponymic heritage is therefore of double nature: 1) forms of toponyms popularized by Austro-Hungarian maps (especially by Spezialkarte) influenced a wide toponymic usus as well as the toponymy of the later maps (especially interwar-Polish military maps) - these popularized forms may have differed from the names used by the local communities, which could have been caused by a surveyor's mistake; 2) the topographic and cartographic materials produced by the Austro-Hungarian institutions are a valuable source for toponomastic research. In the paper, the Austro-Hungarian and interwar-Polish topographic manuals are analysed. These documents defined the way a surveyor had to collect and process geographical names. The examples and possible causes of some Polonized forms occurring in Spezialkarte are discussed. Next, the influence of the Austrian maps on the toponymy of Polish maps is explained. Finally, hilarious examples of cartographic name-copying are given.
\end{abstract}

Keywords: toponyms, geographical names, topographic survey, toponymic language usus, Austria-Hungary, Galicia

\section{Einführung}

Die Epoche der habsburgischen Herrschaft über die österreichischen Teilungsgebiete Polens kennzeichnete sich u. a. durch die schrittweise Ersteinrichtung einer modernen Verwaltung. Hand in Hand damit gingen auch umfangreiche Unternehmungen wie die Durchführung von vier Landesaufnahmen und Erstellung des Katasters, die ihrerseits wiederum als eine Grundlage für eine nie zuvor gesehene Entwicklung der Kartographie des Gebietes dienten, wobei vor allem die 1:75.000 Spezialkarte zu nennen ist.

* The author has received funding from the Polish National Science Centre (Narodowe Centrum Nauki) in the Etiuda 4 doctoral scholarship funding scheme, application no. 2016/20/T/HS2/ 00024. Der Artikel ist im Rahmen eines wissenschaftlichen Aufenthaltes am Institut für Slawistik der Universität Wien entstanden. Der Verfasser dankt Herrn Professor Michael Moser, dem wissenschaftlichen Betreuer des Aufenthaltes, für seine Bemerkungen und Verbesserungen zu dem Text. 
Der vorliegende Artikel gibt einen Überblick über die Einflüsse, welche die oben genannten Maßnahmen auf die schriftliche Fixierung der galizischen Toponymie sowie auf den polnischen toponymischen Sprachgebrauch genommen haben. Die im Artikel thematisierten Aspekte stellen einen Teilaspekt eines größeren Dissertationsprojekts mit dem Titel Uzus toponimiczny. Zarys problematyki teoretycznej (na podstawie polskiej toponimii Huculszczyzny) [Der toponymische Usus. Abriss einer Theorie (am Material der polnischen Toponymie des Huzulenlandes)] dar, das den Prozessen und Mechanismen gewidmet ist, von denen der toponymische Sprachgebrauch gestaltet wird.

\section{Ususformende Kraft der Kartographie}

Hervorgehoben wird hier vor allem die ususformende Kraft der Kartographie (im Sinne der allgemein erhältlichen Kartenwerke), wobei auch ihre Bedeutsamkeit für die Feststellung des ortsüblichen Charakters bestimmter Toponyme in der Vergangenheit besprochen wird. Wichtig ist dabei, dass die Einflüsse der k.u.k. Militärkartographie mit dem Zerfall der Donaumonarchie ebenso wie mit der Ablösung der österreichisch-ungarischen Karten durch eigene Kartenwerke der Nachfolgestaaten keineswegs aufhörten, da das Namensgut $u$. a. der späteren polnischen Militärkarten in vielem auf ihren k.u.k. Vorgängerinnen basierte.

Die Kraft, die Kartenwerke im Bereich der toponymischen Sprachgebrauchsgestaltung aufweisen, beruht in erster Linie auf dem reproduktiven Charakter des toponymischen Usus, d. h. auf der Tatsache, dass Sprachteilhaber v. a. die vorgefundenen Namensverhältnisse wiederholen. Es liegt dabei auf der Hand, dass eine Karte ihre ususgestaltende Kraft v. a. unter den ortsfremden Sprachteilhabern entfaltet, was wiederum direkt mit der Frage der kommunikativen (in diesem Falle auch nominativen) Gemeinschaften und des mentalen bzw. schriftlichen Charakters der Namensgutesspeicherung verbunden ist (s. u.).

Um dies mit einem Beispiel des Einflusses zu illustrieren, den die österreichische Spezialkarte auf die toponymischen Wahlen der polnischen Benutzer der ostkarpatischen Toponymie ausübte, sei hier auf eine Stelle in der von Henryk Gąsiorowski verfassten Rezension eines Werkes von Bolesław Roja mit dem Titel Legioniści w Karpatach w 1914/1915 roku [Die Legionäre in den Karpathen im Jahre 1914/1915] hingewiesen. Gąsiorowski (1878-1947), selbst ein beschlagener Landeskundler, Karpatenkenner und zugleich ein k.u.k. Offizier, hält dem Autor die von ihm begangenen toponymischen Fehler vor und konstatiert:

Ein charakteristischer Zug an der Legionenaktion in den Karpathen, der auch ganz deutlich in dem Werke von Roja auftritt, war es, daß die überwiegende Mehrheit unter der Legionärenkameradschaft mit dem Gebirge überhaupt nicht vertraut gewesen war. In dieser Hinsicht stellte auch der Bataillonskommandant Roja keineswegs eine Ausnahme dar... Es kann daher nicht wundern, daß die österreichische Karte zu einem Vademekum werden musste, an dessen Unfehlbarkeit General Roja mit einem unerschütterlichen, wahrscheinlich schon in der österreichischen Kadettenschule er- 
worbenen, Vertrauen glaubte. Infolgedessen sind auch die Fehler der Karte kritiklos in den Text, vor allem in die Befehle und die Rapporte hineingedrungen (GĄSIOROWSKI 1934: 137). ${ }^{1}$

Ebenso bemerkt Wojciech Krukar, ein Geograph, der sich seit mindestens zwanzig Jahren mit der Mikrotoponymie des Bieszczady- und des Beskid NiskiGebirges beschäftigte und dabei zahlreiche toponomastische Interviews mit den ehemaligen lemkischen und bojkischen Bewohnern dieser Landschaften durchführte, dass eine Karte lokale toponymische Verhältnisse beeinträchtigen und einen lokalen toponymischen Sprachgebrauch stören kann:

...es darf hier eine ungünstige Einwirkung betont werden, die die Karten mit falscher Nomenklatur auf den Informanten haben. [...] Die Autochthonen wiesen auf die Abweichungen in dem auf der Karte fixierten und dem von der Gemeinschaft gebrauchten Namen hin. Manche von ihnen hielten die Karte für eine einzige richtige Quelle des toponymischen Wissens, andere dagegen unterstrichen die Tatsache, dass der auf der Karte vorgefundene Name zu korrigieren sei, denn er sei falsch. ... in Jaśliska... bezeichneten manche Informanten den nordwestlichen Nebenrücken des Berges Kamień als Grabki, denn ,so heißt es auf einer Karte“, früher dagegen sei der Rücken Łozowa genannt worden... (KRUKAR 1992: 48-49). ${ }^{2}$

\section{Toponymische Politik des Wiener Hofes in Galizien}

Im Grunde genommen resultiert das toponymische Erbe der Habsburgermonarchie in Galizien nicht aus einer Benennungs- bzw. Umbenennungsaktivität des Wiener Hofes. Ganz im Gegenteil: die habsburgische Herrschaft in Galizien zeichnete sich durch eine eindeutige Neigung zur Beibehaltung der vorgefundenen Ortsnamen aus - egal wie groß die artikulatorischen bzw. orthographischen Probleme eines deutschsprachigen Ortsnamenbenutzers dann auch sein mochten. So etwa kommen in einem in Lemberg veröffentlichten Schematismus für die Königreiche Galizien und Lodomerien aus dem Jahr 1792 folgende Bezeichnungen bzw. Ortsnamen vor: K. K. Sandezer Kreisamt (43), K. K. Tarnover Kreisamt (45), K. K. Rzeszower Kreisamt (46), Przemisler Kreisamt (49), Zolkiewer Kreisamt (51), Stanislawower Kreisamt (57), Oswiecim (99), Zywiec (100), obwohl es schon früher

1 „Rysem charakterystycznym dla akcji legjonowej w Karpatach, występującym wyraźnie w dziele gen. Roji było także i to, że przeważna część braci legjonowej była $\mathrm{z}$ górami przedtem wogóle nieobeznana. Nie stanowił też wyjątku i sam dowódca bataljonu Roja... Nie można się też dziwić, że wobec tego stać się musiała mapa austrjacka pryncypalnem vademecum z niewzruszoną wiarą wyniesioną przez gen. Roję zapewne jeszcze z austr. szkoły kadeckiej, w jej nieomylność. Wskutek tego też błędy jej dostały się bezkrytycznie do tekstu zwłaszcza w rozkazach i raportach“.

${ }^{2}, \ldots$ warto zwrócić uwagę na niekorzystne oddziaływanie map z błędną nomenklaturą na informatorów. ... autochtoni zwracali uwagę na rozbieżności istniejące między nazwą obiektu zapisana na mapie, a używaną przez społeczeństwo. Niektórzy uważali mapę za jedyne prawdziwe źródło informacji toponimicznej, inni zaś podkreślali, że nazwę, z którą spotkali się na mapie należy poprawić bo jest błędna. ...W Jaśliskach... niektórzy informatorzy określali północno-zachodnie ramię góry Kamień jako Garbki, bo »tak jest na mapach «, dawniej natomiast ramię to zwano Łozowa..." 
deutsche Namen für manche von diesen Städten gegeben hatte. Auf der sog. MiegKarte (s. u.) findet man entsprechend: Tarnow, Rzeszow, Przemysl, Szołkiew (sic!), Stanislawow, Oswieczin und Seibus / Zywiec (aber in dem direkten toponymischen Kontext nur Stary Zywiec). ${ }^{3}$ Generell wurde also die polnische Orthographie beibehalten, jedoch ohne polnische Diakritika. Bemerkenswert ist dabei der Gebrauch der Namensform Oswiecim/Oswieczin (poln. Oświęcim) anstatt Auschwitz und Stanislawow (poln. Stanisławów) - die jedoch später im Laufe des 19. Jhs. zu Stanislau verdeutscht wurde. Bei der Form Szolkiew (auf der Mieg-Karte) handelt es sich um eine falsche „stimmlose“ Anlautsschreibung des Namens Żótkiew $(\check{z}>\check{s}$, wurde aber mit polnischer Orthographie als $s z$ anstatt $s c h$ niedergeschrieben). Solche deutsch-polnischen phonetisch-orthographischen Probleme kommen im Falle der Mieg-Karte sehr oft vor.

Selbst die Tatsache, dass der Name Przemyśl sogar nach der Einrichtung der Festung trotz aller schwer auszusprechenden Zischlauten nie verdeutscht war und dann 1914 und 1915 im Munde der ganzen deutschsprachigen Bevölkerung der Donaumonarchie zu einem richtigen Zungenbrecher wurde, weist auf die namenpolitische Ausrichtung der cisleithanischen Behörden hin. Am Rande darf hier auch erwähnt werden, dass in dem deutschen Exonym Sandez eine frühere polnische, bis zum 18. Jh. lebendige Namensform Sadecz in leicht adaptierter Form tradiert wurde, die später zu $S a c z$ vereinfacht wurde (vgl. RymuT 1987: 166).

\section{Das Wesen des k.u.k. toponymischen Erbes}

Das habsburgische Erbe in der Toponymie Galiziens zeichnet sich durch zwei Merkmale aus: 1) viele Toponyme, die bisher nur lokal bekannt und verwendet worden waren, wurden (vor allem in der 2. Hälfte des 19. Jhs.) - u. a. durch allgemein erhältliche Kartenwerke - weiteren Kreisen zur Verfügung gestellt und zugleich zum ersten Mal kartographisch fixiert; 2) die Resultate der administrativen Maßnahmen, wie die Kataster (Josephinischer und Franziszeischer), die Landesaufnahmen und Kartenwerke, stellen eine höchst wertvolle Quelle für toponomastische Forschungen (vgl. z. B. MRÓZEK 1990: 16-18) sowie für andere Untersuchungen (darunter v. a. geographische und historische; vgl. WOLSKI 2000, STYŚ 1932) dar und können auch bei der Bewertung der vergangenen Verbreitung bestimmter Namensformen in den lokalen Gemeinschaften sehr nützlich sein. Und gerade diese Auswirkungen konstituieren wohl den wichtigsten Aspekt des toponymischen Erbes der k.u.k. Monarchie in Galizien.

Die Bedeutsamkeit des ersten Aspekts, also der kartographischen Fixierung und Verbreitung der Mikrotoponyme, ist enorm. In kleinen kommunikativen Gemeinschaften (etwa von einer Familie oder einem Dorf) hatte es viele Bezeichnungen für kleine Objekte gegeben, die nur diesen kleinen Gemeinschaften bekannt gewesen und von ihnen benannt worden waren. Es hatte sich dabei um einzelne

\footnotetext{
${ }^{3}$ Es wurden die Materialien der Josephinischen Landesaufnahme verwendet, die unter http:// mapire.eu zugänglich sind.
} 
Felder, Weiden, Teile der Wälder usw. gehandelt. Diese Namen waren in der Regel nicht verschriftet gewesen. Es mag dann vielmals dazu kommen sein, dass geographische Namen von ortsfremden k.u.k. Mappeuren ${ }^{4}$ falsch niedergeschrieben wurden und solche verfälschten Namenformen dann durch Kartenwerke in den gröBeren kommunikativen Gemeinschaften verbreitet wurden. Diese kartographisch fixierten Namensformen wurden der Verwaltung, den Touristen, Geographen usw. zur Verfügung gestellt. Das war eine für die Theorie des toponymischen Usus wohl äußerst wichtige Erscheinung: so entstand eine Variation im Sprachgebrauch, die dann reziprok die lokalen toponymischen Verhältnisse beeinflussen kann.

Es liegt auf der Hand, dass es sich bei den österreichischen und später bei den österreichisch-ungarischen Karten in vielen Fällen nicht um eine Erstverschriftung der Namen handelte. Die Kartenwerke haben jedoch generell eine enorme Einwirkung auf den auswärtigen toponymischen Usus (d. h. auf den Sprachgebrauch der größeren kommunikativen Gemeinschaften). ${ }^{5}$ Eine allgemein erhältliche Karte gehört zweifelsohne zu den wichtigsten Faktoren der toponymischen Ususgestaltung.

\section{Galizische Toponyme in der k.(u.)k. Topo- und Kartographie}

Es bietet sich deswegen an, die Wege, die galizische Toponyme zurückgelegt hatten, bevor sie auf die österreichischen Karten gelangten, näher zu besprechen. Die Geschichte der habsburgischen Landesaufnahmen und der Kartographie Galiziens ist in der Literatur, sowohl in der österreichischen wie auch polnischen, gut erfasst worden. $\mathrm{Zu}$ nennen wären hier vor allem die Werke von Ernst Hofstätter, Andrzej Konias und F. P. Faluszczak (HofstäTter 1989, Konias 2000, FaluszcZaK 2011). Eine umfangreiche Besprechung der topographischen Kartographie des westlichen Bojkenlandes in Galizien findet sich bei Jacek Wolski (WOLSKI 2016; dort auch eine umfangreiche Zusammenstellung der - überwiegend polnischsprachigen - Literatur). Peter Jordan analysiert die Einflüsse des Wiener Militärgeographischen Instituts auf die Kartographie der Nachfolgestaaten der k.u.k. Monarchie (JORDAN 2014).

Zu den wichtigsten österreichisch(-ungarischen) topo- bzw. kartographischen Materialien gehören also: die Josephinische Landesaufnahme Galiziens (in der polnischen Literatur v. a. als Mieg-Karte - Mapa Miega - bezeichnet), die von Karl Kummerer von Kummersberg herausgegebene Administrativkarte Galiziens, die Franziszeische Landesaufnahme sowie die Dritte Landesaufnahme und die darauf beruhende, von dem k.(u.)k. Militär-Geographischen Institut in Wien herausgegebene Spezialkarte. Die größte ususgestaltende Kraft weist natürlich das letzte

${ }^{4}$ Mappeur - eine in den analysierten topographischen Instruktionen (s. u.) des Wiener MilitärGeographischen Instituts gebrauchte Bezeichnung für einen Topographen in einem Offiziersrang (vom Hauptmann abwärts) oder im Rang eines Offiziersstellvertreters (IN 1875: 7).

${ }^{5}$ Die für Soziotoponomastik grundlegende Kategorie der kommunikativen Gemeinschaft und der „Reichweite der sprachlichen Speicherung von individuellen Namen“ wurde von Ludwik Zabrocki eingeführt (ZABROCKI 1968). 
der genannten Werke auf. Einen gewissen Einfluss konnte auch die Kummersbergsche Karte ausüben. Faluszczak weist darauf hin, dass die Karte einerseits durch eine Generalisierung der Katasterkarten entstanden war, andererseits als eine gute Grundlage für zahlreiche abgewandelte Karten diente (FALUSZCZAK 2011: 75-76). Die Mieg-Karte und die Materialien der zweiten Franziszeischen Landesaufnahme konnten dagegen den toponymischen Sprachgebrauch kaum beeinflussen, da die Blätter der Mieg-Karte jeweils in nur drei Exemplaren (eine vor Ort gezeichnete Originalausführung und zwei handgezeichnete Kopien) angefertigt und bis zum Jahre 1873 geheim gehalten bzw. schwer zugänglich waren (BUKOWSKI-J ANECZEK 2013; dort auch eine umfangreiche Zusammenstellung der Literatur betreffend die Karte). Die Materialien der zweiten Landesaufnahme blieben unveröffentlicht und dienten als eine Grundlage für nur eine Karte im Maßstab 1:288.000 (FALUSZCZAK 2011: 56). Trotz der fehlenden Ususgestaltungskraft gehören die Mieg-Karte und die zweite Landesaufnahme zu den Kernteilen des habsburgischen toponymischen Erbes in Galizien, weil sie - wie oben erwähnt - als eine wertvolle Quelle für toponomastische Untersuchungen dienen können.

Wegen der enormen toponymischen Bedeutsamkeit der Spezialkarte sollen also die Erhebung, Ermittlung und Bearbeitung des Namensgutes während der dritten Landesaufnahme genauer geschildert werden. Die Aufnahme selbst wurde in den Jahren 1869-1887 durchgeführt und die einzelnen Blätter der darauf basierenden Spezialkarte wurden 1873-1889 bearbeitet (FALUSZCZAK 2011: 79). Die toponomastische Feldarbeit der Mappeure in der dritten Landesaufnahme war v. a. durch die Instruction für die militärische Landesaufnahme (Militärmappirung). II. Theil aus dem Jahre 1875 geregelt (Konias 2000: 92). Und gerade davon, wie diese toponomastische Arbeit von den Mappeuren ausgeführt wurde, hing die Qualität der auf der Spezialkarte vorkommenden Toponymie ab. Im Falle des späteren polnischen Militär-Geographischen Institutes in Warschau (Wojskowy Instytut Geograficzny), das in vielem die Arbeitsprozeduren des Wiener MGIs wiederholte, erklärte T. Czarnota diese toponymische Qualitätsbeziehung folgenderweise:

...die Verantwortlichkeit eines Kartographen für die Lautung eines Namens ist nur eine indirekte. Er erhielt Namen direkt von einem Sprachwissenschaftler, Geographen oder Topographen... und gerade diese sind in erster Linie für die Korrektheit der Benennungen verantwortlich. Eine Pflicht des Kartographen ist es, die entsprechendste Quelle zu wählen, der Umfang seiner Spezialität erlaubt ihm dagegen nicht, auf den Kern der Sache einzugehen (CZARNOTA 1930: 104-105). ${ }^{6}$

Die Bearbeitung der geographischen Benennungen von den Topographen (Mappeuren) im Rahmen der dritten Landesaufnahme und während der Revisionen der Spezialkarte wurde durch mehrere Dokumenten bestimmt, darunter v. a. durch die Instruction für die militärische Landesaufnahme (Militärmappirung).

${ }^{6}$, „...odpowiedzialność kartografa za brzmienie nazwy jest tylko pośrednia. Dostaje on nazwy wprost od językoznawcy, geografa lub topografa... i ci odpowiadają w pierwszym rzędzie za poprawność nazw. Obowiązkiem kartografa jest wybrać najodpowiedniejsze źródło, zakres jego specjalności nie pozwala mu wglądać w meritum zagadnienia“. 
II. Theil aus dem Jahr 1875 (im weiteren als IN 1875 bezeichnet), Instruction für die militärische Landesaufnahme (Militär-Mappirung und Reambulierung). II. Technischer Theil aus dem Jahr 1887 (IN 1887), Anhang A zur Instruction für die militärische Landesaufnahme. II. Technischer Theil aus dem Jahr 1894 (AN 1894) sowie Instruction für die militärische Landesaufnahme. II. Technischer Theil ${ }^{7}$ (2. Ausgabe) aus dem Jahr 1903 (IN 1903). ${ }^{8}$

Eine Vorselektion der Namen, d. h. die Entscheidung, welche der erhobenen Toponyme auf der Karte zu berücksichtigen waren, wurde oft vor Ort von einem Mappeur durchgeführt (IN 1875: 85, IN 1903: 176). Die analysierten Instruktionen betonen generell, wie bedeutsam die Korrektheit des ermittelten Namensguts ist: „....eine richtige und correcte Angabe der Benennungen bleibt eines der wesentlichsten Merkmale der Güte und Verläßlichkeit einer Karte, namentlich einer Karte, die zu Kriegszwecken zu dienen hat" (IN 1875: 59). Diese Erwartung hatte natürlich ganz praktische Gründe: „Namen, welche in einer Gegend weit und allgemein bekannt sind, müssen als Orientirungsmittel jedenfalls aufgenommen werden“" (IN 1875: 91). Diese Korrektheit und in der Tat v. a. die pragmatische Kraft der von einem künftigen militärischen Kartenbenutzer sozusagen mit sich gebrachten Benennungen ist mit der Aufmerksamkeit, die die Instruktionen dem lokalen toponymischen Sprachgebrauch widmeten, eng verbunden.

Bei der Erhebung und Selektion von Namen sollte sich ein Mappeur auch an dem „Kriegszweck“ der Karte orientieren: „....jedoch muß die Auswahl der zu beschreibenden Objecte eine sehr sorgfältige sein und sich nach der militärischen Wichtigkeit derselben richten“ (IN 1875: 93). Zu beschreiben waren also:

...jene einzelnen Höfe und Häuser welche auf freien Höhen liegen, weit sichtbar sind und auch eine große Umsicht gestatten; jene, welche eine tactische Bedeutung haben und in deren Nähe Geschützplacirungen möglich sind, um eine Straße oder ein Defilé zu beherrschen; jene, welche in der Gegend besonders bekannt sind, und als Orientirungsobjecte dienen können; jene, welche an Straßentheilungen, Sättel und Anderen Uebergangspuncten liegen (IN 1875: 93).

Vermeiden sollte man dagegen die Beschreibung der „Culturen“, also der in der Land- und Forstwirtschaft benutzten Terrainflächen wie Felder, Wiesen, Wälder, Weiden (AN 1894: 19). Berücksichtigt man die Tatsache, dass die Spezialkarten zwischen 1888 und 1914 zwei Revisionen erfuhren (JORDAN 2014: 53), die teilweise gemäß der neueren Instruktion (AN 1894) durchgeführt wurden, so kann es auch nicht wundern, dass z. B. ein 1914 ausgeführtes Blatt der Spezialkarte, auf dem das Tschornohora-Gebirge kartiert wurde, deutlich weniger „CulturenNamen“ im Bereich des Hauptrückens des Gebirges beinhaltet als eine 1876 veröffentlichte Version desselben Blattes.

\footnotetext{
${ }^{7}$ Der Verfasser dankt Herrn Professor Peter Jordan und Herrn Dipl.-Ing. Helmut Meckel für die Ermöglichung des Zuganges zu der IN1903.

${ }^{8}$ Die Instruktionen sind in separaten Artikeln ausführlich besprochen worden: die sprachlichen Aspekte der Namensermittlung s. WŁOSKOWICZ 2015a, die graphischen Eigenschaften und die Regeln der Beschriftung s. WŁosKOWICZ 2015b.
} 
Diese scheinbar wenig wesentliche, „Cultur-Namen“ betreffende Empfehlung hatte - v. a. in Verbindung mit dem oben erwähnten militärischen Zweck der Karte - in der Tat schwere Folgen: es wurden die Namen bevorzugt, die gerade nicht die wichtigsten für lokale Gemeinschaften waren. Oft hatte dann ein Mappeur ein taktisch nutzbares Objekt zu benennen, das eigentlich namenlos war, weil es keine wirtschaftliche Bedeutung hatte.

Das im Rahmen der Aufnahme und Revision erhobene Namensgut beeinflusste nicht nur in der späteren Form einer Karte verschiedene sprachliche Texte (wie z. B. landeskundliche Beschreibungen), sondern auch befand sich in gewissem Grade in einem reziproken Verhältnis mit denselben:

Auch sonstige Behelfe, z. B. bereits vorhandene Karten, insbesondere jene der verschiedenen Touristen-Vereine und Clubs, die Reisehandbücher, die topographischen Post-Lexika, die geistlichen Schemas, Orts-Repertorien u. dgl. werden zur Ermittlung einer richtigen Nomenclatur wesentlich beitragen, müssen daher benützt werden; die sich ergebenden Differenzen können Anlass zu mancher Richtigstellung bieten (IN 1887: 174).

Äußerst folgenschwere Bestimmungen betrafen die Auswahl der maßgebenden Informanten:

Da eine fehlerhafte Aussprache der Führer und die Unkenntnis der Landessprache von Seite des Mappeurs oft zu Verwechslungen und Schreibfehlern führen kann, so ist nie zu unterlassen, die politischen Behörden, Förster, Gutsverwalter, Geistliche, Ärzte u. dgl. bei der Richtigstellung und Ergänzung der Nomenclatur zu Rathe zu ziehen (IN 1887: 174).

Generell wurde dem lokalen toponymischen Sprachgebrauch großes Gewicht beigemessen, was v. a. von der oben erwähnten Sorge um die pragmatische Wirksamkeit des später auf einer Karte vorhandenen Namensguts diktiert war. Die Instruktion aus dem Jahre 1903 drückt dies eindeutig aus: „Nur jene Namen, welche der Bevölkerung geläufig sind, haben für den Soldaten Wert" (IN 1903: 167). Die Frage des lokalen Usus war am engsten mit der Frage der sprachlichen Heterogenität der Bevölkerung verbunden. Das Vorgehensweise war in dieser Hinsicht durch die analysierten Richtlinien folgenderweise bestimmt:

Bei Ortschaften, Gebieten ec., welche gemischte Bevölkerung haben und mehrere Namen - in verschiedenen Sprachen - führen, ist der allgemein übliche oder der dem größern Bevölkerungsantheile zukommende als Hauptname zu bezeichnen, der andere jedoch auch beizusetzen (IN 1875: 60).

In gemischtsprachigen Gegenden sind auch die von den verschiedenen Nationalitäten gebrauchten Namen zu erheben (IN 1903: 167).

Bei gemischtsprachiger Bevölkerung ist der am häufigsten gebrauchte Namen vorauszusetzen; die anderen üblichen Bezeichnungen sind in Klammern beizufügen, jedoch nur dann, wenn sie wesentlich anders lauten und von einen bemerkenswerten Theile der Bevölkerung benützt werden (IN 1903: 176). 
Nun wird ein innerer Widerspruch bei der Instruktionen deutlich: einerseits sollte die „dem größern Bevölkerungsantheile zukommende“ Sprachvariante bevorzugt werden, andererseits aber stellten ,,die politischen Behörden, Förster, Gutsverwalter, Geistliche, Ärzte u. dgl.“ eine maßgebende Kontrollinstanz dar. Im Falle Ostgaliziens und des Großteils der galizischen Karpaten (wo zahlenmäßig die ruthenisch- bzw. ukrainischsprachige Bevölkerung der polnischsprachigen überlegen war) führte es dazu, dass auf der Spezialkarte immerhin manchmal auch die polnischsprachige bzw. polonisierte Namensformen vorkommen, wobei es auf der Hand liegt, dass die Mehrheit der lokalen Bevölkerung wohl eine ostslawische Form benützen musste. Bei den entscheidenden „Orts-Repertorien“ waren höchstwahrscheinlich auch vor allem polnischsprachige Namensformen zu finden, was in gewissem Grade die politische Stellung des Polnischen in Galizien in der 2. Hälfte des 19. Jhs. widerspiegelte. Einige Beispiele der auf den älteren Blättern der Spezialkarte (teilweise) polonisierten Namen der geographischen Objekte in den Karpaten sind: Makówka, Bratkowska, Mariszewska wielka, Bystrzec, Góra (als Gattungsname), Kituluwka (später auf der polnischen Karte Kitytówka), Kręta, Stóg (Stoch), ${ }^{9}$ Czarny dit, Biaty Czeremosz, Pisany kamień. Die Polonisierung erfolgte also auf verschiedenen Ebenen: auf einer phonologischen (Бистрець > Bystrzec), phonologischer / lexikalischen (Маківка > Makówka) ${ }^{10}$ lexikalischen (велика $>$ wielka; Білий > Biały; камінь > kamień), phonologisch-orthographischen (Krynta $>$ Kręta). Beachtenswert ist dabei, dass auf der Spezialkarte dialektale huzulische Eigenschaften der Namensformen oft nicht berücksichtigt wurden. So kommt auf der Karte im Huzulenlande etwa standardukrainisches Dit anstatt Git und Żabie anstatt Żebie ${ }^{11}$ vor. ${ }^{12}$

${ }^{9}$ So wurde der Gipfel auf der Karte beschriftet: als Stóg (Hauptname) und Stoch (daneben, in Klammern). Die ukrainische/huzulische Namensform Stih (Cmiz) wurde also ins Polnische übersetzt ( cmic 'Heuhaufen, Heuschober' > stóg) bzw. ans Polnische adaptiert ( $\mathrm{Cmiz}>\mathrm{Stoch}$ ), wobei die Orthographie der Adaptierung auch falsch ist, denn das ukr. Zeichen 2 für $/ \mathrm{h} /$ sollte durch das poln. $h / \mathrm{h} /$ und nicht $c h / \chi /$ ersetzt werden. In der heutigen polnischen Standardsprache ist die Opposition $/ \mathrm{h} /:|\chi|$ offensichtlich neutralisiert, in der damaligen polnischen Aussprache in Ostgalizien war sie dagegen völlig lebendig.

${ }^{10}$ Im Falle der lexikalischen Polonisierung geht es um eine Art „Übersetzung“ eines formengleichen Appellativums, manchmal auch mit einer appellativischen Bedeutungsverschiebung Маківка 'вершина (гори) $\approx$ Gipfel (eines Berges)' > Makówka 'Mohnkapsel'.

${ }^{11}$ Eine dialektale (obwohl keine spezifische) Eigenschaft des Huzulischen ist die Veränderung $t^{\prime}, d^{\prime}>k^{\prime}, g^{\prime}$. Die Veränderung $a>e$ erfolgt nach $\check{c}, \check{s}, \check{z}$ in den osthuzulischen Mundarten und nach allen palatalen Konsonanten in den westhuzulischen Mundarten (HRABEC 1950: 29-30).

${ }^{12}$ Die sprachlichen Eigenschaften sowie der Grad der möglichen Polonisierung der karpatischen Toponyme aus dem Gebiet des Huzulenlandes wurden am Beispiel der Toponymie des galizischen Teiles des Spezialkarte-Blattes Zone 13 Col. XXXI (vom 1876 und 1914), des sich damit vollkommen deckenden Blattes der polnischen Militärkarte Mapa taktyczna Polski (1:100.000) P56 S39 (vom 1933) und der entsprechenden Ausschnitte der Kummersberg-Karte, der zweiten Landesaufnahme sowie einer polnischen touristischen Karte Mapa turystyczna Karpat Polskich (Blatt 3) in einem separaten Artikel analysiert (s. WŁOSKOWICZ 2015c). 


\section{Einflüsse auf den polnischen Sprachgebrauch und die polnische Kartographie}

Die Einwirkung der Spezialkarte auf den polnischen toponymischen Sprachgebrauch u. a. im Bereich der ostkarpatischen Namen war groß. Die Veröffentlichung des Kartenwerkes fiel mit der Entwicklung des polnischen Tourismus in dem Huzulenlande (v. a. im Tschornohora-Gebirge) zusammen. Deutliche toponymische Einflüsse der Karte sind z. B. in einem Reisebericht von M. Turkawski (1880) festzustellen. Dies ist umso eindeutiger, als Turkawski in einer Fußnote hinzufügt, dass die Höhen der Gipfel nach der „spezialen Karte des Generalstabes in Wien“ (,według specyalnéj mapy generalnego sztabu w Wiedniu“) angegeben seien (TuRKAwsKi 1880: 56). Die Blätter der Spezialkarte stellten für die polnischen Touristen noch lange nach dem ersten Weltkrieg das grundlegende Kartenmaterial dar. Sogar die Erstellung der polnischen Militärkarte in der Zwischenkriegszeit (Mapa taktyczna 1:100.000) hatte die toponymische Einwirkung der Spezialkarte nicht beendet, da die polnische Militärkartographie der Zweiten Republik in vielem die früheren k.u.k. Materialien ausnutzte, die gemäß dem Vertrag von Saint-Germain den Nachfolgestaaten überlassen wurden.

Der Ausmaß dieses Nachlasses war enorm:

Aus österreichisch-ungarischer Produktion lagen 1918 für später polnisches Gebiet... 389 Aufnahmesektionen im Maßstab 1:25.000 (1892-1908 berichtigt), 107 Blätter der „Spezialkarte“ 1:75.000 (1888-1917 revidiert), 55 Blätter der „Generalkarte von Mitteleuropa“ 1:200.000 und 11 Blätter der „Übersichtskarte von Mitteleuropa“ 1:750.000 in der Abbildung nach Bonne vor (JORDAN 2014: 60).

Nach der Wiedererstehung Polens im Jahre 1918 wurden die österreichischungarischen Aufnahmesektionen oft als Hilfsmittel bei der Neuaufnahmen verwendet, vor allem aber die habsburgischen Karten revidiert (reambuliert) oder - in den ersten Jahren - einfach nachgedruckt. So wurde von den polnischen Topographen das ursprünglich von ihren k.u.k. Kollegen ermittelte Namensgut verwendet. Die Erhebung und Bearbeitung der „Nomenclatur“ wurde durch die polnischen Instruktionen geregelt, und zwar durch Instruktionen vom 1925 (IN 1925), 1936 (IN 1936) und 1937. Die älteste belehrte den polnischen Topographen, dass:

Die Ermittlung von Namen soll mit besonderer Sorgfalt bei dem Revidieren von russischen und deutschen Karten durchgeführt werden, da auf diesen Karten die Lautung der Namen in sehr vielen Fällen deformiert ist (IN 1925: 167-168). ${ }^{13}$

Die österreichischen Karten wurden nicht erwähnt, und so wurde auch implizit die Qualität ihres Namensgutes anerkannt, wobei auch die Tatsache eine

13 „Ustalanie nazw winno być prowadzone ze szczególną starannością przy rewizji map rosyjskich i niemieckich, gdyż na mapach tych brzmienie nazw jest w bardzo wielu wypadkach zniekształcone“. 
entscheidende Rolle spielen konnte, dass galizische Toponyme auf den k.u.k. Karten generell nicht verdeutscht worden waren. Die neuere polnische Instruktion empfahl dagegen weitgehende allgemeine Vorsicht beim Umgang mit den vorgefundenen Kartenwerken:

Die auf alten Karten vorkommenden Namen sind nicht immer maßgebend und sollen daher als eine Grundlage für die Namensermittlung im Terrain nicht genützt werden, denn sie können nicht mehr verwendet werden oder durch fremde Einflüsse verdorben sein. Die Ermittlung von Namen im Terrain soll ohne jegliche Rücksicht auf alte Karten, Repertorien u. dgl. aufgenommen werden, damit ein reiner, durch sprachliche, graphische oder andere Fehler nicht verdorbener Name erhoben werden kann (IN 1936: 61). ${ }^{14}$

Dass diese Anweisungen von den polnischen Topographen nicht immer eingehalten wurden, ergibt sich aus der im weiteren geschilderten Zusammenstellung der aus den k.u.k. auf die polnischen Karten abgeschriebenen Namen, die falsch (s. u.) sind oder mindestens in den Interviews mit der lokalen Bevölkerung nicht belegt werden konnten.

In seiner neusten Arbeit analysiert der oben schon erwähnte Wojciech Krukar das Namensgut verschiedener Karten des westlichen Bojkenlandes (das ungefähr dem polnischen Bieszczady-Gebirge entspricht), bespricht die toponymischen Beziehungen, die es zwischen diesen Karten gibt, und vergleicht ihre Toponymie mit seinen eigenen Ermittlungen (KRUKAR 2016: v. a. 316-332).

Dass manche Namen aus den österreichisch-ungarischen Karten bzw. Aufnahmesektionen auf die Karten des polnischen MGIs (Wojskowy Instytut Geograficzny) abgeschrieben wurden, liegt auf der Hand.

Die Beweise dafür (aus dem Gebiet des heutigen polnischen Teiles des Bieszczady-Gebirges) gruppiert Krukar nach folgenden Kategorien: 1) Toponyme, die in den Interviews mit den ehemaligen oder zurückgekehrten bojkischen Bewohnern nicht belegt werden konnten (hier nennt Krukar auch mindestens drei Unterkategorien: a) Oronyme [Bergnamen], die durch Transposition eines Oikonyms [Siedlungsnamens] entstanden sind, b) nicht belegte Namen, die dem ortsüblichen Namensgut fremd sind, c) Namen, die zwar in den Interviews in dem [alten] lokalen Sprachgebrauch [eines Dorfes] nicht belegt werden konnten, aber sich ohne weiteres in den regionalen Namenskontext einfügen [d. h. sozusagen endemisch wirken]); 2) belegte Toponyme, die jedoch sowohl auf den MGI-, als auch auf den WIG-Karten in einer deformierten Form vorkommen; 3) Toponyme, die auf den Karten beider Institute falsch lokalisiert wurden; 4) Toponyme, die aus den MGIKarten auf die WIG-Karten falsch abgeschrieben wurden (KRUKAR 2016: 326-331).

14 „Nazwy znajdujące się na starych mapach nie zawsze są miarodajne, przeto nie należy ich używać jako podstawy do ustalania nazw w terenie, ponieważ mogą być już nieużywane lub skażone wpływami obcemi. Do ustalania nazw w terenie należy przystępować bez jakichkolwiek sugestyj, wypływających ze starych map, wykazów i t. p., aby móc wyłowić nazwę czystą, nieskażoną błędami językowemi, pisowniowemi, czy innemi“. 
Die Zusammenstellung der von Krukar angeführten Beispiele der Fehler würde so aussehen:

\begin{tabular}{|c|c|c|c|c|}
\hline \multirow{4}{*}{$\begin{array}{c}\text { 1) nicht belegte } \\
\text { Toponyme }\end{array}$} & $\begin{array}{c}\text { Name auf einer } \\
\text { österreichi- } \\
\text { schen Karte }\end{array}$ & $\begin{array}{c}\text { Name auf einer } \\
\text { WIG-Karte }\end{array}$ & $\begin{array}{c}\text { Die/Der richti- } \\
\text { ge Form/Name }\end{array}$ & Ortschaft \\
\cline { 2 - 5 } & \multicolumn{3}{|c|}{ a) aus der Oikonymie transponiert } \\
\cline { 2 - 5 } & Smerek & Smerek & Wysoka & Wetlina \\
\cline { 2 - 5 } & b) im ortsüblichen regionalen Namensgut nicht vorkommend \\
(deformiert?)
\end{tabular}

Die aus den MGI-Karten auf die WIG-Karten übertragenen Toponyme (KRUKAR 2016: 327-331)

Die angeführten Beispiele mögen jedoch ein falsches Bild von der WIG-Produktion malen. Denn das auf den vom Institut veröffentlichten Kartenwerken vorkommende Namensgut war generell reich und richtig, obwohl Topographen in der Regel wenig Zeit für die toponomastische Arbeit hatten:

Die von den Offizieren-Topographen des [polnischen] Militär-Geographischen Instituts durchgeführte Ermittlung der Namen ist im Allgemeinen gut, doch nicht mangelfrei; es wurde ein großer Fortschritt in Hinsicht des besseren Verstehen der Bedeutsamkeit dieser Sache gemacht. [...] Der Hauptgrund für die Mängel, die noch vorkommen, ist v. a. eine zu kurze Zeit, die einem Topographen für die Kontrolle eines Kartenblattes im Terrain zur Verfügung steht. Hier sind natürlich wichtigere Bedürfnisse entscheidend. Zu knapp sind nämlich unsere Fachkräfte und v. a. die Geldmittel, als dass wir das Land vollständig mappieren könnten. Daher die Eile in der Arbeit 
und hohe Ansprüche, die an die Topographen gestellt werden. [...] Vorwiegend wegen des Zeitmangels fügt ein Topograph nur wenige neue Namen diesen hinzu, die sich schon auf einem Grundblatt befinden, wobei er auch für ihre angemessene Überprüfung oft keine Zeit hat (ROMANOw 1929: 136-137). ${ }^{15}$

\section{6. „Die Macht des Abschreibens“}

Auch während seiner eigenen Feld-Untersuchungen konnte der Verfasser des vorliegendes Artikels ein „kartographisches langes Dauern“ der Toponyme feststellen, die in dem lokalen toponymischen Usus völlig andere Objekte bezeichnen, als es sich aus Karten ergeben mag. Auf einem Bergrücken, der im TschornohoraGebirge die Dörfer Bystrec und Dzembronia voneinander trennt, ist es gelungen, ein paar Dutzend Mikrotoponyme zu erheben, wobei Karten dort generell nur zwei bzw. drei Namenformen für zwei Gipfel aufweisen. Diese sind:

\begin{tabular}{|c|c|c|}
\hline & Gipfel 1 (westlicher) & Gipfel 2 (östlicher) \\
\hline $\begin{array}{c}\text { Josephinische Landes- } \\
\text { aufnahme (Mieg-Karte) }\end{array}$ & Stipainski b. & - \\
\hline $\begin{array}{c}\text { Kummersberg-Karte, } \\
\text { Blatt 51 }\end{array}$ & - & Scepa[ni/in]ski (?) \\
\hline $\begin{array}{c}\text { Franziszeische } \\
\text { Landesaufnahme }\end{array}$ & - & Sciepanski B. \\
\hline $\begin{array}{c}\text { Spezialkarte, 1876, } \\
\text { Zone 13 Col. XXXI }\end{array}$ & Góra piaskowa & Stepański \\
\hline $\begin{array}{c}\text { Spezialkarte, 1914, } \\
\text { Zone 13 Col. XXXI }\end{array}$ & Góra piaskowa & Stepański \\
\hline $\begin{array}{c}\text { Mapa turystyczna } \\
\text { Karpat Polskich, Blatt 3 }\end{array}$ & Góra - Piaskowa & Stepański \\
\hline $\begin{array}{c}\text { Mapa taktyczna Polski } \\
\text { WIG, 1933, Blatt P56 S39 }\end{array}$ & Kosaryszcze & Stepański \\
\hline
\end{tabular}

Der Gipfel 2 ist in der Tat namenlos, Stepanski ist dagegen der Name eines Weilers auf seinem südlichen Hang. Der Name Góra Piaskowa ist den lokalen Informanten nicht bekannt, Koszerysz(cz)e bezeichnet dagegen 1) den ganzen Rücken oder 2) einen ausgedehnten Pass / Nebenrücken südlich des Hauptrückens -

15 „Ustalenie nazw przez oficerów-topografów Wojskowego Instytutu Geograficznego jest naogół dobre, choć nie bez braków; uczyniono ogromny postęp w kierunku większego zrozumienia ważności tej sprawy. [...] Główna przyczyna braków, jakie się jeszcze zdarzają, leży przedewszystkiem w zbyt małym czasie, którym dysponuje topograf do sprawdzania arkusza mapy w terenie. Naturalnie decydują tu potrzeby ważniejsze. Za mało mamy bowiem sił fachowych, a głównie środków finansowych do całkowitego skartowania kraju. A stąd pośpiech w pracy i duże wymagania stawiane topografom. [...] Przeważnie z braku czasu, topograf podaje tylko niewielką ilość nowych nazw miejscowości ponad te, które znajdowały się na arkuszu podstawowym, przyczem na należyte sprowadzenie [es soll heißen: sprawdzenie - W. W.] ich często nie ma czasu“". 
ja nach dem Dorfteil, in dem nach diesem Namen gefragt wurde. Die Namensverteilung, wie sie auf der WIG-Karte vorkommt, ist dann auch auf verschiedenen nach dem Zweiten Weltkrieg ausgeführten Karten zu finden: auf den sowjetischen Stabskarten, den ukrainischen und polnischen Touristenkarten (nach 1991) usw.

Und das ist gerade, sozusagen, das dritte Leben des habsburgischen toponymischen Erbes: das auf den k.u.k. Karten basierende Namensgut der polnischen WIG-Karten wurde nach dem Zweiten Weltkrieg zur Hauptquelle der kartographischen Toponymie. Und wenn man die Tatsache berücksichtigt, dass u. a. in den polnischen Karpaten (im Bieszczady- und Beskid Niski-Gebirge) die lokalen kommunikativen (und toponymischen) Gemeinschaften völlig (Bojken) oder für mehrere Jahren (Lemken) durch das Unternehmen Weichsel (Akcja „Wista“) zerstört wurden, so wird die Bedeutung der von den k.u.k. Topographen geleisteten Arbeit ganz klar.

Die „Macht des Abschreibens“ in der Kartographie führt dann auch zu ganz komischen Situationen: Höchstwahrscheinlich während der dritten Aufnahme, wohl auf der ungarischen Seite der damaligen cis- / transleithanischen Grenze, wurde ein Name Sivakovska dolina für einen Grenzberg nördlich des Łupków-Passes von einem ortsfremden Mappeur niedergeschrieben. Das Toponym ist dann in eine Aufnahmesektion eingedrungen, davon wurde es auf die Spezialkarte übernommen, davon wiederum auf die polnische WIG-Karte, nach dem Zweiten Weltkrieg auf zahlreiche militärische, amtliche und touristische Karten. Und so wird bis heute ein Berg offiziell eigentlich als ein Tal (= poln. dolina) bezeichnet.

\section{Das k.u.k. toponymische Erbe als eine Quelle für toponomastische Forschung}

Wie erwähnt, beruht die Bedeutsamkeit des habsburgischen toponymischen Erbes auch auf der Tatsache, dass die Ermittlungen der k.(u.)k. Mappeure und die österreichisch-ungarischen Kartenwerke bei der Bewertung der früheren lokalen Verbreitung bestimmter Namensformen sehr nützlich sein können.

Ein gutes Beispiel dafür wäre hier der Name des drittgrößten Gipfels des Tschornohora-Gebirges. Ein Streit um den ,richtigen“ Namen ist in Polen in den 30er Jahren entbrannt, was überwiegend damit verbunden war, dass auf dem Berg ein großes astronomisches Observatorium errichtet werden sollte und der Gipfel so auch Schlagzeilen machte. Neben den zwei konkurrierenden Oronymen Czarna Góra und Pop Iwan wurde sogar ein drittes vorgeschlagen: Szczyt Rozśpiewany (etwa Singender Gipfel, was eine Anknüpfung an angebliche Windgesänge sein sollte). Der Name wurde daher zum Thema der wissenschaftlichen und publizistischen Debatte. Piotr Kontny argumentierte für Czarna Góra, dass der Name Pop Iwan von den lokalen Huzulen überhaupt nicht gebraucht werde und dass in den Büchern des Josephinischen Katasters vom 1788 ausschließlich verschiedene orthographische Varianten der Bezeichnung Czarna Góra vorkommen, der Name Pop Iwan ist dagegen nicht zu finden (KonTNY 1936). Auch Stefan Hrabec vertritt eine ähnliche Meinung, wenn er feststellt: „Wohl auch von romantischen Touristen 
wurden die Namen für die Gipfel... und Hänge geschaffen:Pop Iwan ... Zaroślak...“ (HRABEC 1950: 227).

Die Bezeichnung Popiwan muss jedoch der lokalen Bevölkerung am Ende des 18. Jhs. geläufig gewesen sein, da sowohl auf einer Sektion der Josephinischen Aufnahme Ungarns als auch auf einer Sektion der Mieg-Karte der Gipfel ausschließlich Pop Iwan Ruskÿ B. und Pop Iwan heißt. Der Name ist also keine touristische Neuschöpfung. Das ist auch der Fall bei der Bezeichnung Zaroślak, die auf der Kummersberg-Karte in der Form Zaroslek vorkommt und vermutlich aus dem Franziszeischen Kataster übertragen wurde. Der Name ist bekannt geworden, da seit den 70er Jahren des 19. Jhs. dort eine touristische Berghütte funktionierte.

\section{Literatur}

AN 1894 = Anhang A zur Instruction für die militärische Landesaufnahme. II. Technischer Theil. Wien, 1894.

Bukowski-JANECZEK 2013 = Bukowski Waldemar, JANECZEK Andrzej: Mapa józefińska Galicji (1779-1783) w przededniu edycji. Przedmiot i założenia programu wydawniczego. Studia Geohistorica 1 (2013): 91-112.

CZARnOTA 1930 = Czarnota Tadeusz: Polski Przegląd Kartograficzny. T. 1-2. Wiadomości Stużby Geograficznej 1930/1: 99-107.

FaluszCZAK 2011 = FaluszCZAK F. P. Kartografia Galicji Wschodniej. Rzeszów, 2011.

GĄSIOROWSKI 1934 = GĄSIOROWSKI Henryk: Karpaty Wschodnie jako teren walk legjonowych w świeżych publikacjach. Wierchy 12 (1934): 133-140.

HOFSTÄTTER 1989 = HOFSTÄTTER Ernst: Beiträge zur Geschichte der österreichischen Landesaufnahmen. Ein Überblick der topographischen Aufnahmeverfahren, deren Ursprünge, ihrer Entwicklungen und Organisationsformen der vier österreichischen Landesaufnahmen. Bd. 1-2. Wien, 1989.

HRABEC 1950 = HrABEC Stefan: Nazwy geograficzne Huculszczyzny. Kraków, 1950.

IN 1875 = Instruction für die militärische Landesaufnahme (Militärmappirung). II. Theil. Wien, 1875.

IN 1887 = Instruction für die militärische Landesaufnahme (Militär-Mappirung und Reambulierung). II. Technischer Theil. Wien, 1887.

IN 1903 = Instruction für die militärische Landesaufnahme. II. Technischer Theil. Wien, 1903.

IN 1925 = Instrukcja topograficzna. Czesść II techniczna. Warszawa, 1925.

IN 1936 = Instrukcja topograficzna Wojskowego Instytutu Geograficznego. Część III. Zdjęcie stolikowe. Warszawa, 1936.

JORDAN 2014 = JORDAN Peter: Das Wiener Militärgeographische Institut und seine Bedeutung für die Kartographie in Ostmittel- und Südosteuropa. In: 250 Jahre Landesaufnahme. Festschrift. Wien, 2014. 51-73.

KONIAS 2000 = KonIAS Andrzej: Kartografia topograficzna Ślaska Cieszyńskiego i zaboru austriackiego od II połowy XVIII wieku do poczatku XX wieku. Katowice, 2000.

KonTNY 1936 = KontnY Piotr: A więc... Czarna Góra? Niema szczytu „Rozśpiewanego“ ani nawet „Popa Iwana“. Jest nazwa, którą zna tradycja! Ilustrowany Kuryer Codzienny, 10 kwietnia 1936. 20-21.

KRUKAR 1992 = KRUKAR Wojciech: Wybrane nazwy topograficzne na mapach Łemkowszczyzny Wschodniej. Magury'92. Warszawa, 1992. 34-49. 
KRUKAR 2016 = KRUKAR Wojciech: Nazwy terenowe. In: WOLSKI Jacek (Hrsg.): Bojkowszczyzna Zachodnia - wczoraj, dziś i jutro. T. 2. Warszawa, 2016. 271-345.

MrózeK 1990 = MrózeK Robert: System mikrotoponimiczny Śląska Cieszyńskiego XVIII wieku. Katowice, 1990.

ROMANOW 1929 = RoMANOW Wiktor: Organizacja prac nad ustaleniem nazw miejscowości w Polsce. Wiadomości Stużby Geograficznej 1929/3-4: 130-139.

RYMUT 1987 = Rymut Kazimierz: Nazwy miast Polski. Wrocław-Warszawa-KrakówGdańsk-Łódź, 1987.

STYŚ 1932 = STYŚ Wincenty: Metryki gruntowe józefińskie i franciszkańskie jako źródła do historji gospodarczej galicji. In: BuJAK Franciszek, RutKowsKi Jan (Hrsg.): Roczniki Dziejów Społecznych i Gospodarczych. T. 2. Lwów, 1932. 57-92.

TURKAWSKI $1880=$ TURKAWSKI M. A. Wspomnienia Czarnohory. Warszawa, 1880.

WŁosKowicz 2015a = WŁosKowicz Wojciech: Toponomastyczna praca topografa w AustroWęgrzech i w II Rzeczypospolitej. Prace Językoznawcze 2015/3: 137-153.

WŁosKOWICZ 2015b = WŁOSKOWICZ Wojciech: Labels on the maps of the Third Military Survey of Austria-Hungary and on the survey maps of the Military Geographical Institute (Wojskowy Instytut Geograficzny) in Warsaw in the light of survey manuals. Polish Cartographical Review 47 (2015): 31-43.

WŁosKOWICZ 2015c = WŁosKOWICZ Wojciech: Toponimia Huculszczyzny na austro-węgierskich i polskich mapach topograficznych (od poł. XIX w. do 1939 roku). In: VALENTOVÁ Iveta (Hrsg.): 19. slovenská onomastická konferencia. Bratislava, 28-30. apríla 2014. Bratislava, 2015. 298-307.

WOLSKI 2000 = WOLSKI Jacek: Austriacki kataster podatku gruntowego na ziemiach polskich oraz jego wykorzystanie w pracach urzędowych i badaniach naukowych. Polski Przegląd Kartograficzny 32 (2000): 199-212.

WOLSKI 2016 = WOLSKI Jacek: Kartografia topograficzna Bojkowszczyzny Zachodniej (1772-1939). In: WolSKI Jacek (Hrsg.): Bojkowszczyzna Zachodnia-wczoraj, dziś $i$ jutro. T. 1. Warszawa, 2016. 107-174.

ZABROCKI 1968 = ZABROCKI Ludwik: Zasięgi wewnętrznego językowego magazynowania nazw jednostkowych. In: Hrabec S. et al. (Hrsg.) Symbolae philologicae in honorem Vitoldi Taszycki. Wrocław-Warszawa-Kraków, 1968. 416-424. 